\title{
Understanding Treatment Burden for Children Treated for Growth Hormone Deficiency
}

\author{
Meryl Brod $^{1} \cdot$ Lise Højbjerre $^{2} \cdot$ Suzanne Lessard Alolga $^{1} \cdot$ Jane F. Beck $^{1}$ • \\ Lars Wilkinson $^{2} \cdot$ Michael Højby Rasmussen ${ }^{2}$
}

Published online: 6 April 2017

(c) The Author(s) 2017. This article is an open access publication

\begin{abstract}
Objective Growth hormone deficiency (GHD) treatment for children requires growth hormone injections, typically administered daily until the child reaches adult height. Child GHD treatment burden is not well understood and no disease-specific measures exist to assess this burden. The purpose of the study was to explore GHD treatment burden for children and their parents by conducting concept elicitation interviews supporting a theoretical model of the impact of GHD treatment.

Methods Four focus groups (in Germany) and 52 telephone interviews (in the UK and USA) were conducted with children/adolescents with GHD aged 8 to $<13$ years and parents of children with GHD aged $\geq 4$ to $<13$ years. The purpose of the interviews was to understand the experience of GHD treatment from the child's perspective, and for parents, the impact of their child's treatment on themselves. Interview transcripts were analyzed thematically based on modified grounded theory principles.

Results Interviews with 70 respondents who produced descriptions $(n=73)$ of patients experiences with GHD treatment (three parents spoke for two children each) were conducted. Analysis identified three major areas of GHD treatment burden for children: physical; emotional wellbeing; and interference. Parent burdens identified were: emotional well-being and interference. Modifiers such as treatment efficacy and duration, which may impact the degree of treatment burden severity, were identified.
\end{abstract}

Meryl Brod

mbrod@ thebrodgroup.net

1 The Brod Group, 219 Julia Avenue, Mill Valley, CA 94941, USA

2 Novo Nordisk, Søborg, Denmark
Conclusions Overall treatment burden of child GHD is considerable for children and their parents. The concept elicitation and theoretical model can be used to develop a disease-specific outcome measure, which adequately reflects the burden of GHD treatment for children and their parents.

\section{Key Points for Decision Makers}

Treatment burden of growth hormone deficiency (GHD) for children and their parents is substantial.

Study findings indicate treatment burden severity may be modified by the duration of treatment and treatment effectiveness.

Using the results of this study, a GHD diseasespecific outcome measure can be developed to reflect the burden of GHD treatment for children and their parents.

\section{Introduction}

Growth hormone deficiency (GHD) in childhood is evinced by a very slow or flat rate of growth in comparison with other children of the same age [1,2]. A child is considered growth hormone $(\mathrm{GH})$ deficient when $\mathrm{GH}$ levels are low or not present [3]. The prevalence of childhood GHD reported is within the range of 1.8-2.9 per 10,000 [4-6]. A recent study reported the incidence of childhood-onset GHD to be 
2.58 for male individuals and 1.70 for female individuals per 100,000 [7].

Consensus in the medical community is that GH-deficient children require GH replacement therapy [8] to increase growth velocity. If GHD is diagnosed and treated early, relatively normal height and development are usually acquired [1,2], but if GHD is left untreated, or develops late in childhood, shorter-than-average height and delayed puberty may result $[2,9]$. The psychological and behavioral effects of GHD have been reported to include social withdrawal, shyness, anxiousness, and depression [10-17]. Improvement in self-esteem, emotional well-being, and mood in children treated with GHD has been shown [17-19].

Treatment for GHD is by GH injections, typically administered daily until the child reaches adult height [1]. Growth hormone deficiency treatment burden and convenience can be important aspects for treatment adherence because the therapeutic benefits of the $\mathrm{GH}$ treatment are not immediate, and treatment or adherence may be compromised by low motivation. Few studies have been conducted that assess burdens specific to GHD treatment; however, the available literature suggests that GHD treatment can be difficult to manage because of refrigeration and storage burdens, inconvenience when reconstitution is necessary, and managing overnight travel (a major contributor to missed doses) [20]. These burdens are in addition to the pain caused by the injections themselves [20].

Disease-specific measures are known to be more sensitive and have greater responsiveness to change over time with treatment, as the items in the measure are more targeted to the disease population being studied [21]. Unfortunately, no well-validated disease-specific measures currently exist that assess the impact on multiple dimensions [22] of this injectable treatment on children, adolescents, or their parents. The purpose of this study was to explore the burden of GHD treatment for children, adolescents, and their parents and to conduct concept elicitation to develop a GHD treatment impact model, which would have adequate conceptual validity to support the development of disease-specific treatment burden patientreported outcome and observer-reported outcome measures.

\section{Methods}

Focus groups or telephone interviews with GHD children, ages 8 to $<13$ years, and parents of GHD children, ages $\geq 4$ to $<13$ years, were conducted in Germany, the UK, and USA. In Munich, Germany, two focus groups for parents and two focus groups for children were conducted in the native language by a trained local facilitator. Groups with children were divided by sex and led by a same-sex moderator. In the UK and USA, the recruited individuals were too geographically dispersed to gather sufficient numbers in focus groups, so one-on-one telephone calls were conducted; for calls with children, the child was offered a sex-matched interviewer. Interviews in the UK were primarily conducted by a male, native UK Englishlanguage speaker with participants in Manchester, England and Glasgow, Scotland. Interviews in the USA were primarily conducted by a female, native US English-language speaker. Participants in the USA were geographically dispersed around the country. The lead interviewers in the UK and USA were supported by two female and one male USlanguage interviewers to accommodate child preference for sex of interviewer and scheduling needs. A purposive sampling method was used for both focus group and interview selection. A combined written assent/consent form was used to obtain documented assent from all older child/adolescent participants (ages 8-12 years) along with documented consent from their parent/guardian. All parent/guardian (informant) participants provided written consent to participate in focus groups and verbal consent to participate in telephone interviews. This study was approved by the Western Institutional Review Board (Tracking No. 1145991).

Child respondents were eligible if they met the following criteria: (1) pre-pubertal, age 8 to $<13$ years with a diagnosis of isolated GHD, GHD as part of multiple pituitary hormone deficiencies, or organic GHD; (2) a maximum stimulated GH level of $<10 \mathrm{ng} / \mathrm{mL}(\mu \mathrm{g} / \mathrm{L}$ ) on two separate stimulation tests performed either on the same day or on 2 separate days, OR one stimulation test performed along with an insulin-like growth factor-1 test resulting in a maximum stimulated GH level of $<10 \mathrm{ng} / \mathrm{mL}(\mu \mathrm{g} / \mathrm{L})$ and an insulin-like growth factor-1 level two standard deviations below the mean reference range for age and sex; (3) negative signs for intracranial tumor or tumor growth, OR if GHD occurred after treatment for any brain tumor, the patient has to be at least 1 year in clinical remission; and (4) currently receiving any prescription $\mathrm{GH}$ treatment for no more than 12 months, or never treated with GH. Recruitment matched the characteristics of the population, as closely as possible, of the future clinical trial population in which the measure is intended to be used. Growth hormone treatments were pooled together without any distinction between the approved products. Parent/guardian (observer) respondents were eligible if their child met the diagnostic and medical criteria noted above; however, the age of their child could be any age under 13 years. Additionally, parent respondents were required to live in the same residence as the child with GHD and be able to provide information on the child's GHD and treatment, and report on the child's observed behavior. 
Child and parent respondents were excluded from the study if the child had: (1) any clinically significant abnormality likely to affect growth or the physician's ability to evaluate growth as being causally related to GHD, such as, but not limited to, chronic diseases such as renal insufficiency, spinal cord irradiation, and malnutrition; (2) overt diabetes mellitus (fasting blood sugar $>126 \mathrm{mg} / \mathrm{dL}$ ) and impaired fasting sugar (fasting blood sugar $>100 \mathrm{mg} / \mathrm{dL}$ after repeated blood analysis); (3) chromosomal abnormalities and medical syndromes (Turner's syndrome, Laron syndrome, Noonan syndrome, or absence of GH receptors), with the exception of septooptic dysplasia; or (4) congenital abnormalities (causing skeletal abnormalities), Russell-Silver Syndrome, or skeletal dysplasia.

Three recruitment strategies were employed. First, eligible child and adolescent patients were identified by physicians from their current patient caseload. The physician or designated staff person contacted the parent/guardians of eligible patients to determine their, and/or their child's, interest in the study. Once permission was received, their contact information was forwarded to the study for recruitment. Second, national and international GHD-related advocacy and support organizations posted recruitment information on their discussion and social media sites, providing contact information for the study for interested respondents. Last, respondents were identified by professional research organizations that recruit study participants. These organizations contacted individuals enrolled in their proprietary databases and prequalified them by telephone. Participants received an honorarium of US \$125 for in-person focus groups or US $\$ 100$ for telephone interviews.

To guide the interviews, a semi-structured interview guide was designed, based on the literature and discussion with clinical experts, to elicit the perceived symptoms, burden, and impacts of GHD as well as GHD treatment effects on social, physical, and psychological aspects of daily living. Items and probes were designed to be age specific to accommodate child respondents. For children who had been treated for GHD, questions asking about perceived differences pre- and post-treatment were also included. Further, it was expected that young children (under approximately age 8 years) would not be able to complete a patient-reported outcome measure by themselves [23] and that a parent observer-reported outcome measure, and not a proxy measure (reporter responds to what they think/perceive about the child), would be needed. Therefore, it was critical for parents to report only actual and not perceived or presumed impacts on the child. To facilitate observer reports, parents were instructed to answer questions about what they had actually witnessed or been told about by another person as having witnessed. To ensure this was the case, follow-up questions for impacts reported by parents asked parents to report concrete examples of what they had seen or been told that led them to report on the impact. The interview guide evolved iteratively as completed focus groups and interviews were used to guide and inform subsequent discussions. Therefore, issues raised by patients and parent/guardians in earlier groups/interviews were further explored and either confirmed or rejected based on the content of additional group discussions.

Graduate level-trained individuals with backgrounds in qualitative interviewing, focus group moderation, and primary education conducted all interviews, which were facilitated in the native language of the host country. To ensure consistency among interviewers, all interviewers participated in telephone trainings with the US lead interviewer to review the project objectives and the interview guide. Additionally, the lead interviewer listened in to the initial calls of all interviewers to supervise and provide feedback. In Germany, the lead interviewer met with and trained the moderators in person and then observed/listened to the focus groups in person and in real time with simultaneous translation (one group was observed/listened to in real-time via live streaming).

Individual child and parent/guardian telephone interviews were 60-75 min long and in-person focus groups, held at a focus group facility, were $2 \mathrm{~h}$ long. Interviews and focus groups were audio-recorded, transcribed, and translated into English where appropriate.

In line with US Food and Drug Administration guidance on patient-reported outcome development [24], the concept elicitation phase entailed a qualitative study design. Data were qualitatively analyzed through an adapted grounded theory approach, entailing developing and refining a theory based on concepts derived during the research process. Specific techniques included the use of open, axial, selective, and in-vivo coding, the constant comparative method, writing memos, theorizing, exploring particular words and terms for multiple meanings and applications, and negative case analysis [25]. Transcripts were analyzed for content by theme using Dedoose, Version 7.0.21, SocioCultural Research Consultants, LLC, Manhattan Beach, CA, USA, a qualitative data analysis system [26]. A preliminary code list was developed a priori based upon the interview and focus group guide, which served as the basis of the coding tree and was iteratively revised throughout the coding process. A single coding tree was used for all transcripts. Codes were added as new themes emerged during transcript review. When a new code was added, a review of previously coded transcripts occurred to ensure that all occurrences of the new code were captured during the coding process. The transcripts were coded in the chronological order in which the interviews and focus groups occurred and were each reviewed and coded at least three 
times to ensure accuracy and consistency. Themes were then aggregated into larger domains. The analysis was organized by major themes and sub-themes that arose in the interviews. To help determine which impacts were the most commonly experienced, the coding captured the number of child and parent/guardian participants who reported each treatment burden impact.

Based on findings from the qualitative analysis, a conceptual model of major and minor treatment burden impacts was developed. To be included in the model, the impact needed to be a discrete rather than a broad descriptor of the impact (e.g., worry rather than general dislike) and could potentially be affected by treatment. Major impacts were those reported by $15 \%$ or more of the study sample and a minor impact if reported by less than $10 \%$ of the sample. Items that were endorsed by between 10 and $<15 \%$ of the sample were individually examined and included as major if they were endorsed by $20 \%$ of parents or children, were proximal rather than distal impacts, not solely related to height, and/or considered conceptually important by clinical experts interviewed (for concepts that clinicians would be aware of) or in the literature. All major impacts were confirmed as both relevant and important by respondents during the cognitive debriefing interviews (conducted after the item generation was completed with an independent sample of parents and children).

\section{Results}

\subsection{Sample Description}

Thirty-nine children, aged 8 to $<13$ years, with GHD and 31 parents of children, aged $\geq 4$ to $<13$ years, with GHD participated $(n=70)$. Children were aged $8-9$ years $(n=7)$ and 10 to $<13$ years $(n=32)$. Of the 31 parents, 19 were parents of child respondents who participated in the study. Fifty respondents $(71.4 \%)$ were recruited directly from clinical contacts, four (5.7\%) from professional research organization patient panels and 16 (22.9\%) from advocacy and support organizations. Parent respondents provided descriptions of children under the age of 8 years $(n=14)$, aged $8-9$ years $(n=8)$, and aged 10 to $<13$ years $(n=12)$. Data were collected in Germany in four focus groups ( $n=19$ respondents) and in the UK and USA by individual telephone interviews $(n=51)$. Three parents were interviewed about two of their children with GHD (one in the US, two in Germany). Therefore, for analytic purposes, there were 73 narrative descriptions of GHD.

Of the GHD descriptions gathered by interview or focus group, slightly over half were from US respondents $(n=40,54.8 \%)$ with additional respondents from Germany $(n=21,28.8 \%)$ and the UK $(n=12,16.4 \%)$.
Table 1 presents the breakdown of respondents by country and age of child.

The mean age at GHD diagnosis was 7 years (range $3-12$ years); however, the average age at diagnosis was lowest in Germany (4.6 years) as compared with the UK (7.0 years) and USA (9.4 years). A majority of children had taken GH therapy $(n=66,90.4 \%)$ with treatment beginning on average in Germany at a younger age (4.9 years) than in the UK (7.9 years) or USA (9.5 years). As a result, the average length of time on treatment varied considerably with the longest time on treatment reported in Germany (43.0 months), followed by the UK (7.9 months) and USA (6.5 months). Respondents reported additional health conditions; the most frequent were ear, nose, and throat conditions; lung diseases or other respiratory conditions; and mental health conditions. Table 2 presents the details on the health and demographic characteristics of children and parents associated with the 73 narrative descriptions.

\subsection{Domains and Themes Generated by Telephone Interviews and Focus Groups}

A total of 55 concepts related to the burden of GHD treatment in children emerged from all interviews and focus groups conducted; 36 of these concepts were addressed by participants in both the child and parent/guardian samples. Thematic saturation was separately assessed for the 39 children and 34 parents/guardians in the order in which the interview or focus group occurred. A total of 40 concepts were discussed during the child interviews; after the 17th child interview, $80 \%$ of these concepts had been discussed, and by the 27th interview, $95 \%$ of these concepts had been covered. A total of 51 concepts were discussed during the parent/guardian interviews; after the 15th parent/guardian interview, $80 \%$ of these concepts had been discussed, and by the 25 th interview, $95 \%$ of these concepts had been covered. The three treatment burden domains identified for children were physical, emotional well-being, and interference. The two treatment burden domains identified for parents were emotional well-being and interference. In the following, data for parent/guardian (observer) and child-provided descriptions are combined.

\subsubsection{Physical Treatment Burdens}

Parent and child-provided descriptions of physical treatment burdens focus primarily on the mode of delivery by injection. Their descriptions include injection pain $(n=30,41 \%)$; bruising at the injection site $(n=15$, $21 \%)$; burning, stinging, or soreness $(n=14,19 \%)$; and bleeding from the injection $(n=6,8 \%)$. Many of these 
Table 1 Summary of parent and child participants by country and age (years) of child

\begin{tabular}{|c|c|c|c|c|c|c|c|}
\hline \multirow[t]{2}{*}{ Country } & \multicolumn{2}{|c|}{ Child age 4 to $<8$ years } & \multicolumn{2}{|c|}{ Child age $8-9$ years } & \multicolumn{2}{|c|}{ Child age 10 to $<13$ years } & \multirow{2}{*}{$\begin{array}{l}\text { No. of GHD descriptions } \\
(N=73) ; n(\%)\end{array}$} \\
\hline & Child & Parent & Child & Parent & Child & Parent & \\
\hline Germany & & 6 & 4 & 4 & 7 & & $21(28.8)$ \\
\hline UK & & 4 & 1 & 1 & 6 & & $12(16.4)$ \\
\hline USA & & 4 & 2 & 3 & 19 & 12 & $40(54.8)$ \\
\hline Total & & 14 & 7 & 8 & 32 & 12 & $73(100)$ \\
\hline
\end{tabular}

GHD growth hormone deficiency

impacts were amplified by the frequency and efficacy of treatment, the confidence/skills of the person administering the injection, and whether or not the child self-administers injections.

Physical Aspects: Child

Parent: "It hurts. Yes, I mean she screams. She cries it hurts."

Child: "Sometimes when I tense up, it hurts a lot, and I usually hold on to my leg because I don't want it to hurt a lot."

Descriptions included more frequent reports of injection pain in boys and bruising at the injection site in girls. Physical impacts such as injection pain, and burning, stinging, or soreness were much less frequently reported for children under the age of 8 years. Table 3 presents the breakdown within the physical treatment burdens domain.

\subsubsection{Emotional Well-Being Treatment Burdens}

Parent- and child-provided descriptions of emotional impacts suggest that there is a wide range of emotional response to GHD treatment, which suggested in broad terms, a general dislike of treatment $(n=25,34 \%)$ or being upset $(n=12,16 \%)$ with treatment. Specific emotional burdens include fear of injections ( $n=27,37 \%)$, worry $(n=22,30 \%)$, embarrassment about treatment ( $n=14,19 \%$ ), unhappiness about frequency of injections ( $n=13,18 \%)$, annoyance about injections $(n=11,15 \%)$, and feeling different from other children because of treatment ( $n=11,15 \%)$. Parent and child respondents report, when children worry, they worry about missing doses ( $n=11,15 \%)$, injection administration $(n=9,12 \%)$, and injection pain $(n=4,5 \%)$. For many respondents, the emotional impact was stronger at treatment initiation. For example, injection fear at treatment initiation was noted in close to one third of the narrative descriptions $(n=21$, $29 \%$ ), with much lower frequency of injection fear lasting past the initiation of treatment and remaining unresolved $(n=10,14 \%)$. Treatment efficacy, experienced as increased growth, was an important modifier for the reduction of emotional impact over time, as was the duration of treatment.

Emotional well-being: Child

Parent: "We haven't made it to all of the eight sites yet. So it seems like when it's a new site, he gets worried."

Child: "I just like feel worried. Like I don't want to miss a shot, but I may need to if all my spots are bruised."

Incidences of child acceptance of treatment $(n=13$, $18 \%$ ), not being particularly bothered by treatment ( $n=32,44 \%$ ), or the child actively wanting and liking their GHD treatment ( $n=23,32 \%$ ) were also reported by parent and child respondents. Acceptance and desire for treatment was often linked to the child's desire to grow and the obvious and visible improvements with treatment.

Descriptions of boys included more frequent reports of acceptance and wanting or liking GHD treatment, but boys also were more frequently reported to fear injections, worry, and feel nervous when preparing for injections. Descriptions of girls included more frequent reports of feeling annoyed by GHD treatment. For children under the age of 8 years, acceptance and nervousness when preparing for injections was reported more frequently and unhappiness with the frequency of treatment reported less frequently than for the other age groups. Both liking treatment and feeling different from other children was reported less frequently for children aged 8-9 years, and feeling upset was reported more frequently for children aged 8-9 years than for the other age groups. Disliking treatment by injection was reported less frequently for children aged 10 to $<13$ years, and this age group also experienced a high rate of acceptance of treatment.

Parents self-reported being emotionally impacted themselves by GHD treatment for their children. They noted their own worry $(n=21,62 \%)$, including worry about treatment administration $(n=20,59 \%)$, causing pain to their child ( $n=13,38 \%$ ), and medication costs 
Table 2 Health and demographic characteristics of children with growth hormone deficiency (GHD) described in the study

\begin{tabular}{|c|c|c|c|c|}
\hline Demographic characteristics & Germany $(n=21)$ & $\mathrm{UK}(n=12)$ & USA $(n=40)$ & Total $(N=73)$ \\
\hline \multicolumn{5}{|l|}{ Age group counts, years, $n(\%)$} \\
\hline 4 to $<8$ & $6(28.6)$ & $4(33.3)$ & $4(10.0)$ & $14(19.2)$ \\
\hline $8-9$ & $8(38.1)$ & $2(16.7)$ & $5(12.5)$ & $15(20.5)$ \\
\hline 10 to $<13$ & $7(33.3)$ & $6(50.0)$ & $31(77.5)$ & $44(60.3)$ \\
\hline \multicolumn{5}{|l|}{ Sex, $n(\%)$} \\
\hline Female & $8(38.1)$ & $4(33.3)$ & $10(25)$ & $22(30.1)$ \\
\hline Male & $13(61.9)$ & $8(66.7)$ & $30(75)$ & $51(69.9)$ \\
\hline \multicolumn{5}{|l|}{ Ethnicity, $n=52, n(\%)$} \\
\hline White & Not collected $^{\mathrm{a}}$ & $10(83.3)$ & $36(90.0)$ & $46(88.5)^{\mathrm{a}}$ \\
\hline Persian & & $1(8.3)$ & & $1(1.9)^{\mathrm{a}}$ \\
\hline Asian & & $1(8.3)$ & & $1(1.9)^{\mathrm{a}}$ \\
\hline Other & & & $4(10.0)$ & $4(7.7)^{\mathrm{a}}$ \\
\hline \multicolumn{5}{|l|}{ Household income, US\$ } \\
\hline Less than 20,000 & $2(9.5)$ & $1(8.3)$ & $1(2.5)$ & $4(5.5)$ \\
\hline $20,001-40,000$ & $2(9.5)$ & $3(25.0)$ & $2(5.0)$ & $7(9.6)$ \\
\hline $40,001-60,000$ & $1(4.8)$ & $3(25.0)$ & $5(12.5)$ & $9(12.3)$ \\
\hline $60,001-80,000$ & $2(9.5)$ & $1(8.3)$ & $6(15.0)$ & $9(12.3)$ \\
\hline $80,001-100,000$ & $4(19.0)$ & $1(8.3)$ & $13(32.5)$ & $17(23.3)$ \\
\hline More than 100,000 & $3(14.3)$ & $3(25.0)$ & $9(22.5)$ & $13(17.8)$ \\
\hline Decline to answer & $7(33.3)$ & & $4(10.0)$ & $14(19.2)$ \\
\hline \multicolumn{5}{|l|}{ Other prescription medications, $n(\%)$} \\
\hline Yes & $4(19.0)$ & $2(16.7)$ & $23(57.5)$ & $29(39.7)$ \\
\hline \multicolumn{5}{|l|}{ Age at diagnosis, years } \\
\hline Mean (range) & $4.56(3-8)$ & $7.01(3-12)$ & $9.36(3-12)$ & $6.98(3-12)$ \\
\hline \multicolumn{5}{|l|}{ Ever taken GHD therapy } \\
\hline Yes, $n(\%)$ & $20(95.2)$ & $11(91.7)$ & $35(87.5)$ & $66(90.4)$ \\
\hline \multicolumn{5}{|l|}{ Age first started GHD therapy } \\
\hline Mean (range) & $4.87(4-8)$ & $7.89(8-12)$ & $9.47(3-12)$ & $7.41(3-12)$ \\
\hline \multicolumn{5}{|l|}{ Duration (months) of GHD therapy } \\
\hline Mean (range) & $43.0(1-96)$ & $7.9(2-12)$ & $6.5(0.2-16)$ & $17.8(0.2-96)$ \\
\hline \multicolumn{5}{|l|}{ Other health conditions } \\
\hline Arthritis, rheumatic diseases, musculoskeletal conditions & & & $1(2.5)$ & $1(1.4)$ \\
\hline Ear, nose, and throat conditions & $4(19.0)$ & & $6(15.0)$ & $10(13.7)$ \\
\hline Eye disorders & $1(4.8)$ & $1(8.3)$ & & $2(2.7)$ \\
\hline Kidney disease, urinary conditions & & $1(8.3)$ & $1(2.5)$ & $2(2.7)$ \\
\hline Lung disease, respiratory conditions (including allergies and asthma) & $2(9.5)$ & & $15(37.5)$ & $17(23.3)$ \\
\hline Mental health conditions (including depression and anxiety) & & & $13(32.5)$ & $13(17.8)$ \\
\hline Metabolic conditions (including elevated cholesterol) & $2(9.5)$ & $1(8.3)$ & $1(2.5)$ & $4(5.5)$ \\
\hline Stomach, intestinal, gastrointestinal disease & & $1(8.3)$ & & $1(1.4)$ \\
\hline Stroke, neurological condition & & $1(8.3)$ & $2(5.0)$ & $3(4.1)$ \\
\hline Other condition & $1(4.8)$ & $4(33.3)$ & $4(10)$ & $9(12.3)$ \\
\hline None & $13(61.9)$ & $6(50.0)$ & $15(37.5)$ & 34 (46.6) \\
\hline
\end{tabular}

${ }^{\mathrm{a}} n=52$; ethnic identifications were not collected in Germany owing to ethics standards in Germany

$(n=5,15 \%)$. They also reported feeling sadness about the need for treatment $(n=7,21 \%)$, guilt $(n=6,18 \%)$, and frustration with injection administration $(n=5,15 \%)$. Table 4 presents the breakdown within the emotional wellbeing treatment burdens domain.

\subsubsection{Interference Treatment Burdens}

Parent- and child-provided descriptions suggested that the experience of interference resulting from injection treatments is variable. Over half of respondents $(n=41,56 \%)$ 
Table 3 Physical treatment burdens domain by subtheme

\begin{tabular}{|c|c|c|c|c|c|c|}
\hline \multirow[t]{2}{*}{ Physical treatment burdens domain } & \multicolumn{2}{|c|}{ Total narrative descriptions } & \multicolumn{2}{|c|}{ Child-provided descriptions } & \multicolumn{2}{|c|}{ Parent-provided descriptions } \\
\hline & $N=73$ & $\%$ & $n=39$ & $\%$ & $n=34$ & $\%$ \\
\hline Injection pain & 30 & 41 & 21 & 54 & 9 & 26 \\
\hline No problem with injection pain & 14 & 19 & 10 & 26 & 4 & 12 \\
\hline Bruising & 15 & 21 & 9 & 23 & 6 & 18 \\
\hline Burning/stinging/soreness & 14 & 19 & 9 & 23 & 5 & 15 \\
\hline Bleeding & 6 & 8 & 2 & 5 & 4 & 12 \\
\hline
\end{tabular}

Table 4 Emotional well-being treatment burdens domain by subtheme

\begin{tabular}{|c|c|c|c|c|c|c|}
\hline \multirow[t]{2}{*}{ Emotional well-being treatment burdens domain } & \multicolumn{2}{|c|}{ Total narrative descriptions } & \multicolumn{2}{|c|}{ Child-provided descriptions } & \multicolumn{2}{|c|}{ Parent-provided descriptions } \\
\hline & $N=73$ & $\%$ & $n=39$ & $\%$ & $n=34$ & $\%$ \\
\hline Child not bothered by injections & 32 & 44 & 18 & 46 & 14 & 41 \\
\hline Fear of injections & 27 & 37 & 11 & 28 & 16 & 47 \\
\hline Injection fear at initiation/treatment & 21 & 29 & 9 & 23 & 12 & 35 \\
\hline Injection fear ongoing, past initiation/treatment & 10 & 14 & 2 & 5 & 8 & 24 \\
\hline Generally dislikes treatment by injection & 25 & 34 & 13 & 33 & 12 & 35 \\
\hline Child wants/likes treatment & 23 & 32 & 11 & 28 & 12 & 35 \\
\hline Worry (child) & 22 & 30 & 11 & 28 & 11 & 32 \\
\hline Worry about missing doses & 11 & 15 & 6 & 15 & 5 & 15 \\
\hline Worry about injection administration & 9 & 12 & 5 & 13 & 4 & 12 \\
\hline Worry about pain & 4 & 5 & 2 & 5 & 2 & 6 \\
\hline Worry about growth (does not want change) & 1 & 1 & 0 & 0 & 1 & 3 \\
\hline Nervous preparing for injections & 15 & 21 & 4 & 10 & 11 & 32 \\
\hline Embarrassed by treatment & 14 & 19 & 6 & 15 & 8 & 24 \\
\hline Embarrassed only at initiation & 3 & 4 & 2 & 5 & 1 & 3 \\
\hline No problem with embarrassment & 21 & 29 & 8 & 21 & 13 & 38 \\
\hline Unhappy about frequency of injections & 13 & 18 & 7 & 18 & 6 & 18 \\
\hline Acceptance of injections & 13 & 18 & 7 & 18 & 6 & 18 \\
\hline Upset about injections & 12 & 16 & 2 & 5 & 10 & 29 \\
\hline Annoyance about injections & 11 & 15 & 9 & 23 & 2 & 6 \\
\hline Feeling different from other children & 11 & 15 & 8 & 21 & 3 & 9 \\
\hline Emotions increased with treatment & 8 & 11 & 1 & 3 & 7 & 21 \\
\hline Increased fear of doctors and medical procedures & 5 & 7 & 1 & 3 & 4 & 12 \\
\hline Sadness about injections & 2 & 3 & 2 & 5 & 0 & 0 \\
\hline \multicolumn{7}{|l|}{ Emotional impacts on parents } \\
\hline Parent worry & & & & & 21 & 62 \\
\hline Worry about treatment administration & & & & & 20 & 59 \\
\hline Worry about causing pain to child & & & & & 13 & 38 \\
\hline Worry about medication cost & & & & & 5 & 15 \\
\hline Parent sadness & & & & & 7 & 21 \\
\hline Parent guilt & & & & & 6 & 18 \\
\hline Parent frustration & & & & & 5 & 15 \\
\hline
\end{tabular}

noted that treatment interfered with overnight sleepovers or other travel activities. There were also two aspects of interference with overnight travel. First, overnight travel interfered with dose timing and often resulted in deferred/ missed injections $(n=29,40 \%)$. Second, injection administration was disruptive to the experience of 
overnight travel ( $n=20,27 \%)$ because of the need to plan and accomplish the logistics of administration away from home. Additional interference was described for the time needed for children to prepare emotionally for the injection ( $n=20,27 \%$ ), and the need to stop or interrupt daily life activities to accommodate the treatment schedule $(n=17$, 23\%). Potential modifiers for these burdens included duration of treatment, age of treatment start, and whether or not the responsibility for treatment administration was shared with the child.

Interference: Child

Parent: "So a lot of times he won't stay at a friend's house because I can't just go, give him the shot, and come home, or if he's spent the day there and wants to stay, I have to run that over, do it, come back home, so sometimes it's just easier just to go get him, bring him home, and just be done."

Child: "I sleep over at my friend's house sometimes or they sleep over here, but I don't take my shot on those days because they might come and I don't really want to bring the shot with me over there."

Some respondents did report that treatment generally did not interfere with daily activities for them $(n=26,36 \%)$, or that GHD treatment requires a learning curve and that administering injections gets easier over time $(n=26$, $36 \%)$, or becomes routine and part of daily life $(n=25$, $34 \%)$.

Descriptions of boys included more frequent reports of the initial learning curve. Descriptions of girls included more frequent reports of the need to stop and interrupt daily life activities to accommodate the treatment schedule. Reports that treatment administration did not interfere with daily life were more frequent for children ages 10 to $<13$ years than for the younger age groups. Interference with overnight sleepovers or travel was reported less frequently for children under the age of 8 years.

Parents self-reported being impacted themselves by interference resulting from GHD treatment. Half of parents $(n=17,50 \%)$ noted that GHD treatment interferes in family travel and travel planning/logistics. Approximately one third of parents $(n=11,32 \%)$ noted that it took time to prepare their child for the injection and that this was also interfering, and that GHD treatment interferes with their daily and social life $(n=4,12 \%)$. Table 5 presents the breakdown within the interference treatment burdens domain.

\subsubsection{Social Context of Treatment}

Social impacts did not meet the criteria for major impacts as they were determined to be distal and secondary to emotional impacts. Although a few children were fearful that they might be teased or were embarrassed by treatments, there were no reports of teasing or bullying specific to injection treatment by peers or other references to social burdens of treatment in these descriptions. However, a few themes suggest the social context surrounding treatment, much of which is supportive. Many parent and child respondents noted that the child is comfortable telling others about their injections $(n=32,44 \%)$ and a subset of this group indicated that the child often educated peers about his/her injection treatments $(n=22,30 \%)$. In contrast to this, some children preferred to keep their treatments private and did not tell others about them $(n=20$, 27\%). Last, some children experienced social support specifically directed towards their treatments and injections from family or friends $(n=16,22 \%)$. This social support tends to boost their confidence and motivate their attitude towards treatment.

Descriptions of boys included more frequent reports of educating friends and family about their injection treatments. Reports of educating friends and family were less frequent for children under the age of 8 years. Social support for injection treatments was less frequently noted for children aged 8-9 years. Table 6 presents the breakdown within the social aspects of treatment domain.

\subsubsection{Theoretical Model}

The model presents both the proximal and distal treatment burden domains and subdomains for both children and their parents along with key modifiers that can impact the individual experiences with treatment (Fig. 1). For a major domain to be included in the model, it had to be endorsed by both children and parents as important and relevant. Table 7 presents parent and child quotes for each major impact.

\section{Discussion}

Injections are often a challenging mode of medication delivery for many, and GHD treatment is no exception. This qualitative study provides evidence that the experience of GHD treatments is considerable but variable and can be modified by factors such as duration and efficacy of treatment, age, and sex. As with most treatments, adaptation occurs and many of the children and parents who experience difficulty at the initiation of treatment adapt over time and treatment can become a part of their daily routine with reduced or no burden associated with it. However, for some it continues to be a source of physical and emotional pain, and interference in daily life. Thus, the challenge may be to not only reduce burden for the 
Table 5 Interference treatment burdens domain by subtheme

\begin{tabular}{|c|c|c|c|c|c|c|}
\hline \multirow[t]{2}{*}{ Interference treatment burdens domain } & \multicolumn{2}{|c|}{$\begin{array}{l}\text { Total narrative } \\
\text { descriptions }\end{array}$} & \multicolumn{2}{|c|}{$\begin{array}{l}\text { Child-provided } \\
\text { descriptions }\end{array}$} & \multicolumn{2}{|c|}{$\begin{array}{l}\text { Parent-provided } \\
\text { descriptions }\end{array}$} \\
\hline & $N=73$ & $\%$ & $n=39$ & $\%$ & $n=34$ & $\%$ \\
\hline Overnight or other travel activities & 41 & 56 & 23 & 59 & 18 & 53 \\
\hline Overnight activities interfere with dose timing: missed or deferred treatments & 29 & 40 & 18 & 46 & 11 & 32 \\
\hline Treatment interferes with overnight activities (is disruptive) & 20 & 27 & 8 & 21 & 12 & 35 \\
\hline Treatment does not interfere with daily activities & 26 & 36 & 13 & 33 & 13 & 38 \\
\hline Treatment requires a learning curve and it gets easier over time & 26 & 36 & 15 & 38 & 11 & 32 \\
\hline Treatment is routine/part of daily life & 25 & 34 & 7 & 18 & 18 & 53 \\
\hline Time needed to prepare emotionally for injection & 20 & 27 & 4 & 10 & 16 & 47 \\
\hline Needing to stop/interrupt what you are doing for the injection & 17 & 23 & 9 & 23 & 8 & 24 \\
\hline Needing to remember (preoccupation) & 5 & 7 & 5 & 13 & 0 & 0 \\
\hline \multicolumn{7}{|l|}{ Interference for parents } \\
\hline Interferes with family travel & & & & & 17 & 50 \\
\hline No problem with travel & & & & & 7 & 21 \\
\hline Time needed to prepare injection (logistics) or prepare child for injection & & & & & 11 & 32 \\
\hline Interferes with daily and social life & & & & & 4 & 12 \\
\hline Interrupted sleep & & & & & 3 & 9 \\
\hline Accessing medication & & & & & 3 & 9 \\
\hline
\end{tabular}

Table 6 Social aspects of treatment domain by subtheme

\begin{tabular}{|c|c|c|c|c|c|c|}
\hline \multirow[t]{2}{*}{ Social aspects of treatment domain } & \multicolumn{2}{|c|}{$\begin{array}{l}\text { Total } \\
\text { narrative } \\
\text { descriptions }\end{array}$} & \multicolumn{2}{|c|}{$\begin{array}{l}\text { Child- } \\
\text { provided } \\
\text { descriptions }\end{array}$} & \multicolumn{2}{|c|}{$\begin{array}{l}\text { Parent- } \\
\text { provided } \\
\text { descriptions }\end{array}$} \\
\hline & $N=73$ & $\%$ & $n=39$ & $\%$ & $n=34$ & $\%$ \\
\hline Child thinks it is okay for others to know about their injections and tells others & 32 & 44 & 16 & 41 & 16 & 47 \\
\hline Child often finds him/herself educating family and friends about injection treatments & 22 & 30 & 13 & 33 & 9 & 26 \\
\hline $\begin{array}{l}\text { Child keeps treatment private-does not tell others or have injection in front of others (besides } \\
\text { parents/guardians) }\end{array}$ & 20 & 27 & 8 & 21 & 12 & 35 \\
\hline Child experiences social support for injection treatments & 16 & 22 & 9 & 23 & 7 & 21 \\
\hline
\end{tabular}

population for which it continues to be problematic but also to shorten the time until adaption to treatment occurs and/ or reduce the degree of treatment burden during the initial stages of the treatment process.

This study explored the burdens specific to GHD treatment, which few studies have previously researched. Our study findings are comparable to an Internet survey-based study by Kremidas et al. [20], which surveyed patients with GH disorders currently using a prescription GH medication aged 13+ years and caregivers of GH disorder patients currently assisting with using a prescription GH medication, to evaluate issues including administration practices and the reasons behind missed injections. They found that managing overnight travel is a major contributor to missed doses. Our analysis revealed that travel interfered with dose timing and often resulted in deferred/missed injections. As similarly found in the Kremidas et al. article, our study found with regard to interference with travel, the burden of storage of medication was a primary concern of most parents as refrigeration is required.

Another factor previously studied is discomfort and pain experienced from the injection of GH. One study found that caregivers were more likely to agree that injections were painful, and patients were more likely to disagree (though most of those disagreeing patients were adults) [20]. In our study, injection pain and bruising were the top two physical treatment burdens reported by both children and parent/guardians (observer). More than half of children reported injection pain while just over one quarter of parents reported pain. These results must be evaluated in the context that the parents/guardians reported as observers with instruction to report on what they had actually witnessed or been told about by another person as having witnessed. 


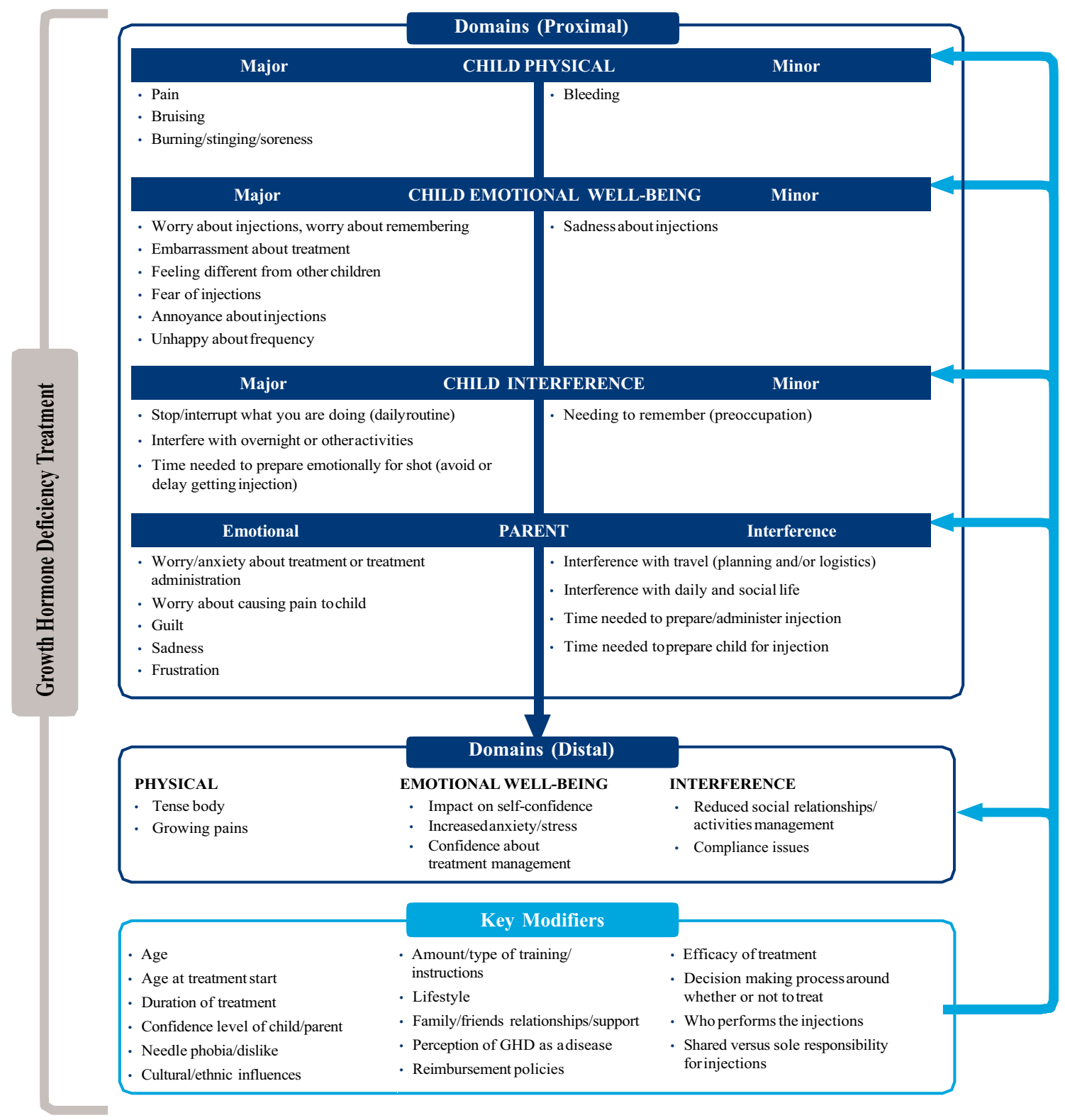

Fig. 1 Preliminary theoretical model of the treatment burden measure-child growth hormone deficiency (GHD)

Additionally, parent- and child-provided descriptions of the negative impact of treatment had some notable similarities. General dislike of treatment and worry were similarly reported by parents and children, but a higher frequency of parents reported their child experienced fear of injections, nervousness preparing for injections, upset about injections, and were embarrassed by treatment. Additionally, interference of GHD treatment with overnight or other travel activities and needing to stop/interrupt daily life activities to accommodate the treatment schedule was reported similarly by both child and parent respondents. Further, parents and children agreed that treatment requires a learning curve and gets easier over time.
According to the criteria for defining a burden as major, there were no major social burdens associated directly with GHD treatments noted by children or parents.

Parents self-reported being substantially impacted themselves by worry surrounding treatment administration, but also by worry about causing the child pain and the medication costs. Feelings of sadness about the need for treatment, guilt, and frustration with injection administration were additional emotions reported. Parents self-reported that the child's treatment interfered with their own lives. Half of parents noted that GHD treatment interfered in family travel and travel planning/logistics and approximately one third of parents noted that the time it took to 
Table 7 Selected quotes for major themes

\begin{tabular}{ll} 
Theme/subtheme & Selected quote \\
\hline
\end{tabular}

Physical aspects: child

Injection pain

Bruising

Burning/stinging/soreness

Emotional well-being: child

Fear of injections

Child worry

Embarrassment

Unhappy about frequency

Annoyance

Feeling different from other children

Emotional well-being: parent

Parent worry

Parent sadness

Parent guilt

Parent frustration
Parent: It hurts. Yes, I mean she screams. She cries - it hurts

Child: Sometimes when I tense up, it hurts a lot, and I usually hold on to my leg because I don't want it to hurt a lot

Parent: She does bruise sometimes from her shot. She is very self-conscious about her bruising, especially when it comes to swimming, because everyone can see it

Child: Sometimes we really can't find a spot because sometimes everything is bruised

Parent: He says it burns

Child: Sometimes it will sting a lot

Child: But sometimes when it hurts a lot, then the spot will bruise a little and that's when it feels a little sore

Child: Straight after I have it maybe it feels a bit numb or a bit sore

Parent: He says: I'm afraid. I'm really afraid. This is very true. He is very insistent

Child: Well, that's when I got pretty scared like about the injection every single night. I was scared that it would hurt a ton

Parent: We haven't made it to all of the eight sites yet. So it seems like when it's a new site, he gets worried

Child: I just like feel worried. Like I don't want to miss a shot, but I may need to if all my spots are bruised

Parent: He doesn't like it. He didn't want anybody to know ... he didn't want anybody to know. So he never talks about it with anybody

Child: I don't want them to know that I get jabs every night because I am afraid they might laugh at me

Parent: Getting a shot every day. That can't be easy on a kid

Child: The worst thing is that I have to get an injection every night. I really don't like that

Parent: Well, I think he is mostly annoyed about the fact that we discuss this now at all. He would rather not have to deal with that topic. He has kind of an avoidance strategy. And now, there is a clear indicator - there is an injection, and there are growth hormones, and he is supposed to grow. And now he has to deal with that a bit more, which he doesn't want to. I think this means a lot of work to him; it is tiresome

Child: But then sometimes I just really don't want to do it, but I have to, so it's kind of annoying sometimes

Parent: He notices that he is different somehow

Child: It just makes me feel different. It's just it's not normal for other kids to have to take shots that I'm friends with

Parent: So I'm trying to not let him see that I'm freaking out because he needs to see me calm. Yeah, it's been hard, too. I get worried, too

Parent: Our new normal was going to include daily injections and the difficulty and the hurt. Every day I would make my kid hurt. You do everything you can in your whole life to make your kids not hurt. That's the tough part

Parent: So it is upsetting because you obviously want your child to be well and healthy and not to go through this

Parent: So you know I'm thankful to doctors and medicine, but there definitely is a part of me that's angry and sad for her

Parent: Because I'm trying to impart on him the importance of getting it, and I'm sure at 12 that's not what he's taking from it. I'm sure he's taking from it that I didn't do it again, and I'm sorry. He has apologized before, and I feel tremendous guilt

Parent: Once he knew it wasn't as bad as he thought in his head, but he was still taking a really long time to prep himself, it began to become frustrating for me 
Table 7 continued

\begin{tabular}{|c|c|}
\hline Theme/subtheme & Selected quote \\
\hline \multicolumn{2}{|l|}{ Interference: child } \\
\hline Overnight or other activities & $\begin{array}{l}\text { Parent: So a lot of times he won't stay at a friend's house because I can't just go, give him } \\
\text { the shot, and come home, or if he's spent the day there and wants to stay, I have to run that } \\
\text { over, do it, come back home, so sometimes it's just easier just to go get him, bring him } \\
\text { home, and just be done } \\
\text { Child: I sleep over at my friend's house sometimes or they sleep over here, but I don't take } \\
\text { my shot on those days because they might come and I don't really want to bring the shot } \\
\text { with me over there }\end{array}$ \\
\hline $\begin{array}{l}\text { Time needed to prepare emotionally for } \\
\text { injection }\end{array}$ & $\begin{array}{l}\text { Parent: Probably the easiest part is once he allows me to go ahead and do it is to just do it. } \\
\text { The prep part is probably the worst because of his having to get himself emotionally ready } \\
\text { Child: That I sometimes simply don't want to do it .... I just run away somewhere }\end{array}$ \\
\hline $\begin{array}{l}\text { Needing to stop/interrupt what you are doing } \\
\text { for injection }\end{array}$ & $\begin{array}{l}\text { Parent: She'll stay out as late as she can, even if she's at her friend's she'll be the last one } \\
\text { and want to stay until the last minute. So sometimes she comes home and she's really tired } \\
\text { and would love to curl up in her bed, but unfortunately she's got to get organized } \\
\text { Child: I just want to do it real fast, so then I can go plan again }\end{array}$ \\
\hline \multicolumn{2}{|l|}{ Interference: parent } \\
\hline Interferes with family travel & $\begin{array}{l}\text { Parent: If you go away, you gotta make sure you bring everything with you, so we don't do } \\
\text { that too often }\end{array}$ \\
\hline $\begin{array}{l}\text { Time needed to prepare injection (logistics) or } \\
\text { prepare child for injection }\end{array}$ & $\begin{array}{l}\text { Parent: I don't know, just doing it in general. I just think it ruins-I feel like it ruins every } \\
\text { single night like it just-until after that and then when he's on cloud nine, I'm like, "What } \\
\text { was I even stressed out about for the last hour trying to figure out exactly the time I was } \\
\text { going to do it?" }\end{array}$ \\
\hline Interferes with daily and social life & $\begin{array}{l}\text { Parent: I will give it a little later if need be, but I always try to just change our plans to leave } \\
\text { what we're doing to come home. [...] It can be rough, but you know you have to adapt for } \\
\text { your kids. [...] I would say (it happens) every other week at least }\end{array}$ \\
\hline
\end{tabular}

prepare their child for the injection, with some parents reporting GHD treatment impacted their daily and social life. Therefore, the impacts of treatment on the parents themselves are not inconsequential.

The concept of treatment burden may not take into consideration the positive benefits of treatment. For example, the incidences of child acceptance of treatment, not being particularly bothered by treatment, or the child actively wanting and liking their GHD treatment were reported by parent and child respondents. However, the purpose of the measure under development has a targeted focus to assess burden and while the benefits of treatment may modulate the burden, they are not in and of themselves central to the assessment of burden.

Understanding the impact of GHD treatment on children and their parents can improve communications between them and their physicians. Through an understanding of GHD treatment burden, physicians can better support parents and children by providing adequate education on medication administration and support for those with continued difficulty with injections. The demonstrated efficacy of treatment and its effect of reducing related disease burdens in emotional, social, and daily life indicates that advocacy by physicians for early treatment of GHD may alleviate the overall burden of the condition that may cause harm. Additionally, ongoing research and development in the improved ease of use of delivery devices and long-acting GH preparations are anticipated to further reduce treatment burden.

As with all research, there are some limitations to this study. First, although this study included reports from 73 child and parent-provided descriptions of GHD experience (a large sample size for qualitative research), these findings may not be generalizable to all children with GHD. Further, it is possible that volunteers for participation in research form a unique population that results in a sample that may not be representative of all children with GHD or their parents. However, given that recruitment employed three different methodologies, the sample pool was potentially broadened.

Additionally, although the interview script prompted for both prospective and retrospective information with the assessment of domains now vs. before individuals started treatment, we attempted to minimize recall bias for those who were taking treatment by having a relatively short duration of time (no more than 12 months) of treatment.

Finally, for some domains and subdomains, the frequency of report was different for children under the age of 8 years. These distinctions may be owing to the fact that parent respondents were the only respondents for this age group, in addition to circumstances associated with being that young. Finally, the child focus groups seemed to have 
a strong cohort effect with little diversity in opinion or experience. While it may be true that the experience of GHD occurs uniformly across these respondents, it also may be true that children in groups tend towards agreement with one another and that the individual interviews were more able to elicit a wider range of experience.

\section{Conclusions}

Overall, GHD treatment burden in children is substantial as is demonstrated in this study. There are modifiers to the experienced severity of treatment burden that may be amenable to interventions such as treatment efficacy, age of treatment initiation, and duration of treatment. Accurate and reliable assessment of treatment burden for both children and their parents may help clinicians to understand better compliance issues and may improve the quality of doctor-patient communications. The concept elicitation and theoretical model can be used to develop a disease-specific outcome measure, which adequately reflects the burden of GHD treatment for children and their parents.

Acknowledgements The authors would like to acknowledge and thank the sites, physicians, patients, and parent/guardians of children with GHD who participated in the study. Without their contributions, the study could not have been successful. We thank Natasa Savic, MD $\mathrm{PhD}$, Knud Vad, PhD, and Johan Adalsteinsson, MD (Novo Nordisk) for their review and input into the manuscript.

Author contributions MB contributed to the research design, analysis, and manuscript preparation; SA contributed to the research design, literature review, manuscript preparation, and conducted focus groups; JFB contributed to the study design and conduct, and manuscript preparation; LW, LH, and MHR contributed to the research design and manuscript preparation.Compliance with Ethical Standards

Funding This study was funded by Novo Nordisk A/S.

Conflict of interest Dr. Brod, Ms. Alolga, and Ms. Beck are advisors/paid consultants to Novo Nordisk. Mr. Wilkinson, Dr. Højbjerre, and Dr. Højby Rasmussen are employees of Novo Nordisk.

Ethics approval All procedures performed in studies involving human participants were in accordance with the ethical standards of the institutional and/or national research committee and with the 1964 Helsinki Declaration and its later amendments or comparable ethical standards.

Consent to participate A combined written assent/consent form was used to obtain documented assent from all older child/adolescent participants (ages 8-12 years) along with documented consent from their parent/guardian. All parent/guardian (informant) participants provided written consent to participate in focus groups and verbal consent to participate in telephone interviews.

Open Access This article is distributed under the terms of the Creative Commons Attribution-NonCommercial 4.0 International License (http://creativecommons.org/licenses/by-nc/4.0/), which permits any noncommercial use, distribution, and reproduction in any medium, provided you give appropriate credit to the original author(s) and the source, provide a link to the Creative Commons license, and indicate if changes were made.

\section{References}

1. Society for Endocrinology. You and your hormones: endocrine conditions. Childhood onset growth hormone deficiency. Reviewed 2014. Available from: http://www.yourhormones.info/endocrine_ conditions/childhood_onset_growth_hormone_deficiency.aspx. Accessed 12 Mar 2016.

2. Medline Plus. Growth hormone deficiency: children. Updated 9 Sep 2013. Available from: http://www.nlm.nih.gov/medlineplus/ ency/article/001176.htm. Accessed 20 Dec 2013.

3. The Child Growth Foundation. Growth hormone deficiency: a guide for parents and patients; Series No: 2. 2003. Available from: http://www.childgrowthfoundation.org/CMS/FILES/02_ Growth_Hormone_Deficiency.pdf. Accessed 12 Mar 2016.

4. Thomas M, Massa G, Craen M, et al. Prevalence and demographic features of childhood growth hormone deficiency in Belgium during the period 1986-2001. Eur J Endocrinol. 2004;151(1):67-72.

5. Vimpani GV, Vimpani AF, Lidgard GP, et al. Prevalence of severe growth hormone deficiency. $\mathrm{Br}$ Med $\mathrm{J}$. 1977;2(6084):427-30.

6. Lindsay R, Feldcamp M, Harris D, et al. Utah Growth Study: growth standards and the prevalence of growth hormone deficiency. J Pediatr. 1994;125:29-35.

7. Stochholm K, Gravholt CH, Laursen T, et al. Incidence of GH deficiency: a nationwide study. Eur $\mathrm{J}$ Endocrinol. 2006;155(1):61-71.

8. Laron Z. Growth hormone therapy: emerging dilemmas. Pediatr Endocrinol Rev. 2011;8(4):364-73.

9. Jovinelly J. Growth hormone deficiency. Healthline Networks, Inc. Updated 24 July 2012. Available from: http://www. healthline.com/health/growth-hormone-deficiency?toptoctest= expand. Accessed 9 Jan 2014.

10. Skuse D, Gilmour J, Tian CS, Hindmarsh P. Psychosocial assessment of children with short stature: a preliminary report. Acta Paediatr Suppl. 1994;406:11-6 (discussion 17).

11. Stabler B. Impact of growth hormone (GH) therapy on quality of life along the lifespan of GH-treated patients. Horm Res. 2001;56(Suppl. 1):55-8.

12. Stabler B, Clopper RR, Siegel PT, et al. Academic achievement and psychological adjustment in short children: the National Cooperative Growth Study. J Dev Behav Pediatr. 1994;15(1):1-6.

13. Abbott D, Rotnem D, Genel M, Cohen DJ. Cognitive and emotional functioning in hypopituitary short-statured children. Schizophr Bull. 1982;8(2):310-9.

14. Sartorio A, Conti A, Molinari E, et al. Growth, growth hormone and cognitive functions. Horm Res. 1996;45(1-2):23-9.

15. Wheeler PG, Bresnahan K, Shephard BA, et al. Short stature and functional impairment: a systematic review. Arch Pediatr Adolesc Med. 2004;158(3):236-43.

16. Sandberg DE, Bukowski WM, Fung CM, Noll RB. Height and social adjustment: are extremes a cause for concern and action? Pediatrics. 2004;114(3):744-50.

17. Abe S, Okumura A, Mukae T, et al. Depressive tendency in children with growth hormone deficiency. J Paediatr Child Health. 2009;45(11):636-40.

18. Geisler A, Lass N, Reinsch N, et al. Quality of life in children and adolescents with growth hormone deficiency: association with growth hormone treatment. Horm Res Paediatr. 2012;78(2):94-9. 
19. Chaplin JE, Kriström B, Jonsson B, et al. Improvements in behaviour and self-esteem following growth hormone treatment in short prepubertal children. Horm Res Paediatr. 2011;75(4):291-303.

20. Kremidas D, Wisniewsky T, Divino VM, et al. Administration burden associated with recombinant human growth hormone treatment: perspectives of patients and caregivers. J Pediatr Nurs. 2013;28(1):55-63.

21. Brod M, Perwien A, Adler L, et al. Conceptualization and assessment of quality of life for adults with attention-deficit/hyperactivity disorder. Prim Psychiatry. 2005;2(6):58-64.

22. Cramer JA, Simeoni MC, Robitail S, et al. Development of a growth hormone injection questionnaire for adolescents. J Adolesc Health. 2004;34(1):27-9.

23. Matza LS, Patrick DL, Riley AW, et al. Pediatric patient-reported outcome instruments for research to support medical product labeling: report of the ISPOR PRO good research practices for the assessment of children and adolescents task force. Value Health. 2013;16(4):461-79.

24. U.S. Dept. of Health and Human Services, Food and Drug Administration, Center for Drug Evaluation and Research, Center for Biologics Evaluation and Research, Center for Devices and Radiological Health. Guidance for industry: patient-reported outcome measures, use in medical product development to support labeling claims. 2009. https://www.fda.gov/downloads/ Drugs/GuidanceComplianceRegulatoryInformation/Guidances/ UCM193282.pdf. Accessed 15 Nov 2016.

25. Corbin J, Strauss A. Basics of qualitative research: grounded theory procedures and techniques, 3rd ed. Los Angeles, CA: Sage; 2008.

26. Dedoose. Version 7.0.21. Manhattan Beach (CA): SocioCultural Research Consultants, LLC; 2016. 